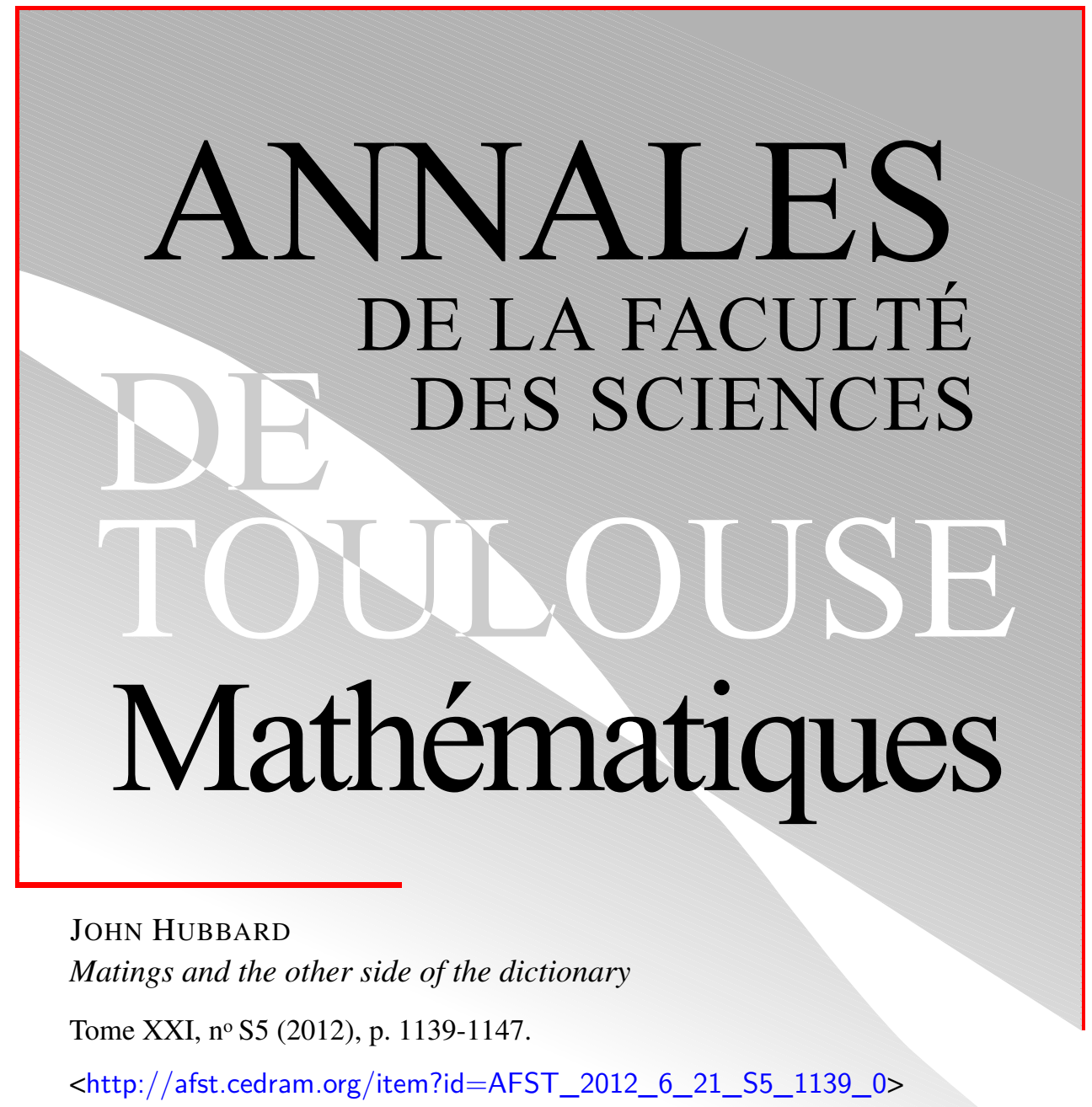

C Université Paul Sabatier, Toulouse, 2012, tous droits réservés.

L'accès aux articles de la revue «Annales de la faculté des sciences de Toulouse Mathématiques » (http://afst.cedram.org/), implique l'accord avec les conditions générales d'utilisation (http://afst.cedram. org/legal/). Toute reproduction en tout ou partie de cet article sous quelque forme que ce soit pour tout usage autre que l'utilisation à fin strictement personnelle du copiste est constitutive d'une infraction pénale. Toute copie ou impression de ce fichier doit contenir la présente mention de copyright.

\title{
cedram
}

Article mis en ligne dans le cadre du

Centre de diffusion des revues académiques de mathématiques

http://www.cedram.org/ 


\title{
Matings and the other side of the dictionary
}

\author{
JoHN HubBard
}

\begin{abstract}
In the theory of rational maps an important role is played by matings. These are probably the best understood of all rational functions, but they are bizarre, and involve gluing dendrites together to get spheres carrying Peano curves.

In the theory of Kleinian groups, there is a parallel construction, the construction of double limits, that is central to Thurston's hyperbolization theorem for 3-manifolds that fiber over the circle with pseudo-Anosov monodromy. It also involves gluing dendrites and Peano curves.

Clearly these two constructions form one entry of the Sullivan dictionary. This article attempts to spell out the similarities and differences.
\end{abstract}

RÉSUMÉ. - Les accouplements forment une classe essentielle d'applications rationelles, sans doute celle qui est la mieux comprise. Mais elle fait intervenir des objets bizarres: recollements de dendrites, courbes de Peano, etc.

La construction analogue pour les groupes Kleiniens est celle des limites doubles. Cette construction est essentielle pour l'hyperbolisation des variétés de dimension trois fibrées sur le cercle.

Ces deux constructions se correspondent par le dictionnaire de Sullivan. Cet article essaie de montrer les similitudes et les différences.

\section{Introduction: the Sullivan dictionary}

Sullivan's dictionary pairs statements about 1-dimensional complex dynamics and statements about 3-dimensional hyperbolic geometry. For instance 


\section{Dynamical systems}

\author{
Julia set \\ non-attracting cycles \\ Yoccoz inequality \\ No wandering domains \\ Tuning \\ Topological characterization of \\ rational functions \\ Parabolic blow-ups
}

\section{Hyperbolic geometry}

Limit set

Non-elliptic fixed points

Bers compactness

Ahlfors finiteness

Klein-Maskit combination

Thurston's hyperbolization theorem

for Haken manifolds

Geometric limits

In some cases, the constructions on the two sides look similar although the proofs are different; for instance, the proof of the density of fixed points in the limit set for Kleinian groups using the convex hull in hyperbolic 3space has no known analog in proving the density of repelling cycles in the Julia set.

In other cases the actual details of the proofs are amazingly similar even though the statements sound unrelated; this is particularly true of Sullivan's No-wandering Domains theorem, whose proof was modeled on the Ahlfors finiteness theorem. It is also the case for the Yoccoz inequality and the compactness of Bers slices: both depend on bounds on the modulus of an annulus embedded in a torus. Further, Thurston's theorem on the Topological Characterization of Rational Functions was modeled after his Hyperbolization Theorem for Haken Manifolds.

There is a glaring gap in the dictionary: there is no known analog on the dynamical side of hyperbolic space $\mathbb{H}^{3}$. Thurston claims that this is the greatest weakness of the theory.

In this article I will look at the entries

\section{Dynamical systems}

Matings

\section{Hyperbolic geometry}

Double limit theorem

In this case, the constructions are clearly parallel, but the known proofs are quite different. Maybe they don't need to be; a familiarity with one side of the dictionary may inspire new and better proofs on the other.

There is nothing really new here, but there is a lack of communication between the two fields and many practitioners of one are unaware of the other. 


\section{The two settings}

\subsection{The mating of polynomials}

Let $p_{1}, p_{2}$ be two polynomials of the same degree $d$ with connected filledin Julia sets $K_{1}, K_{2}$. Choose Böttcher coordinates

$$
\phi_{1}: \mathbb{C}-K_{1} \rightarrow \mathbb{C}-\overline{\mathbb{D}} \text { and } \phi_{2}: \mathbb{C}-K_{2} \rightarrow \mathbb{C}-\overline{\mathbb{D}} .
$$

When $d=2$, the Böttcher coordinate is unique, but when $d>2$ there are $d-1$ choices, and they matter: the mating may exist for some and not for others.

Let $\widetilde{\mathbb{C}}_{1}, \widetilde{\mathbb{C}}_{2}$ be two copies of the complex plane compactified by adding a circle at infinity, adding a point $t_{\infty, i} \in \mathbb{R} / \mathbb{Z}$ at $\lim _{r \rightarrow \infty} \phi_{i}^{-1}\left(r e^{2 \pi i t}\right)$. Both $p_{1}$ and $p_{2}$ extend to maps

$$
\tilde{p}_{1}: \widetilde{\mathbb{C}}_{1} \rightarrow \widetilde{\mathbb{C}}_{1} \text { and } \quad \tilde{p}_{2}: \widetilde{\mathbb{C}}_{2} \rightarrow \widetilde{\mathbb{C}}_{2}
$$

that map $t_{\infty, i}$ to $d t_{\infty, i}$.

Construct a topological 2-sphere

$$
S_{p_{1}, p_{2}}=\left(\widetilde{\mathbb{C}}_{1} \sqcup \widetilde{\mathbb{C}}_{2}\right) / \sim
$$

where $\sim$ identifies $t_{\infty, 1}$ to $-t_{\infty, 2}$.

This sphere carries no natural complex structure, but it does have a map to itself which is $\tilde{p}_{1}$ on $\widetilde{\mathbb{C}}_{1}$ and $\tilde{p}_{2}$ on $\widetilde{\mathbb{C}}_{2}$; these maps are compatible with the gluing and induce a ramified covering map

$$
\tilde{f}_{p_{1}, p_{2}}: S_{p_{1}, p_{2}} \rightarrow S_{p_{1}, p_{2}}
$$

of degree $d$. The space $S_{p_{1}, p_{2}}$ together with the map $\tilde{f}_{p_{1}, p_{2}}$ is called the formal mating of $p_{1}, p_{2}$; it actually depends also on the choices of Böttcher coordinates.

The map $\tilde{f}_{p_{1}, p_{2}}$ is compatible with the equivalence relation $\approx_{p_{1}, p_{2}}$ on $S_{p_{1}, p_{2}}$ defined as follows: it is the smallest equivalence relation with closed equivalence classes, such that for all $t \in \mathbb{R} / \mathbb{Z}$, all points of $\phi_{1}^{-1}\left(r e^{2 \pi i t}\right)$ are equivalent to all points of $\phi_{2}^{-1}\left(r e^{-2 \pi i t}\right)$.

As such, $\tilde{f}_{p_{1}, p_{2}}$ induces a map

$$
f_{p_{1}, p_{2}}: \bar{S}_{p_{1}, p_{2}} \rightarrow \bar{S}_{p_{1}, p_{2}},
$$

where $\bar{S}_{p_{1}, p_{2}}=S_{p_{1}, p_{2}} / \approx_{p_{1}, p_{2}}$. 
There is another way of thinking about $\bar{S}_{p_{1}, p_{2}}$, that is far easier to visualize. This way applies only to the case when $K_{1}$ and $K_{2}$ are locally connected. There are then Caratheodory loops $\gamma_{i}: \mathbb{R} / \mathbb{Z} \rightarrow \partial K_{i}$, and the space $\bar{S}_{p_{1}, p_{2}}$ is the quotient of $K_{1} \sqcup K_{2}$ obtained by identifying $\gamma_{1}(t)$ with $\gamma_{2}(-t)$. The resulting space and map is often called the topological mating of $p_{1}$ and $p_{2}$.

This space $\bar{S}_{p_{1}, p_{2}}$ may appear very bizarre and rather scary, especially when $K_{1}$ and $K_{2}$ are dendrites: $\bar{S}_{p_{1}, p_{2}}$ is obtained by gluing these dendrites to each other.

There is a reassuring theorem of R. L. Moore that asserts that if the equivalence classes do not separate the sphere, are not the whole sphere, and if the quotient space is Hausdorff, then the quotient space is homeomorphic to the sphere, and the quotient map can be approximated by homeomorphisms. The hypotheses of Moore's theorem are often satisfied by $\approx_{p_{1}, p_{2}}$, and the mating construction yields beautiful examples of Moore quotients. But they are still pretty bizarre: each of the dendrites maps surjectively to the quotient sphere, as does the equator of $S_{p_{1}, p_{2}}$, which is then a Peano curve in $\bar{S}_{p_{1}, p_{2}}$.

We say that $p_{1}$ and $p_{2}$ are mateable if $\bar{S}_{p_{1}, p_{2}}$ is homeomorphic to a sphere, and if this sphere carries a complex structure such that $f_{p_{1}, p_{2}}: \bar{S}_{p_{1}, p_{2}} \rightarrow$ $\bar{S}_{p_{1}, p_{2}}$ is analytic; it is then automatically conjugate to a rational function of degree $d$.

Note that in this case where $p_{1}$ and $p_{2}$ are mateable, we have a surjective continuous map $\gamma: \mathbb{R} / \mathbb{Z} \rightarrow \mathbb{P}^{1}$ (a Peano curve) giving a commutative diagram

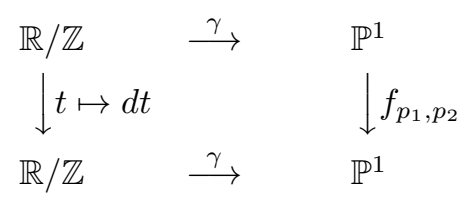

So $\gamma$ semiconjugates the complicated map $f_{p_{1}, p_{2}}$ to $t \mapsto d t$; it somehow unwinds the sphere like a ball of yarn, turning it into a circle, and the complicated rational function $f_{p_{1}, p_{2}}$ unwinds to the simple mapping $t \mapsto d t$.

In dynamical systems, the main question is: to what extent does the statement that $\bar{S}_{p_{1}, p_{2}}$ is homeomorphic to a sphere imply that $p_{1}$ and $p_{2}$ are mateable, and to what extent is the resulting mating unique.

There are many results in this direction: the present volume is largely devoted to this question, and I will not pursue it further here. 

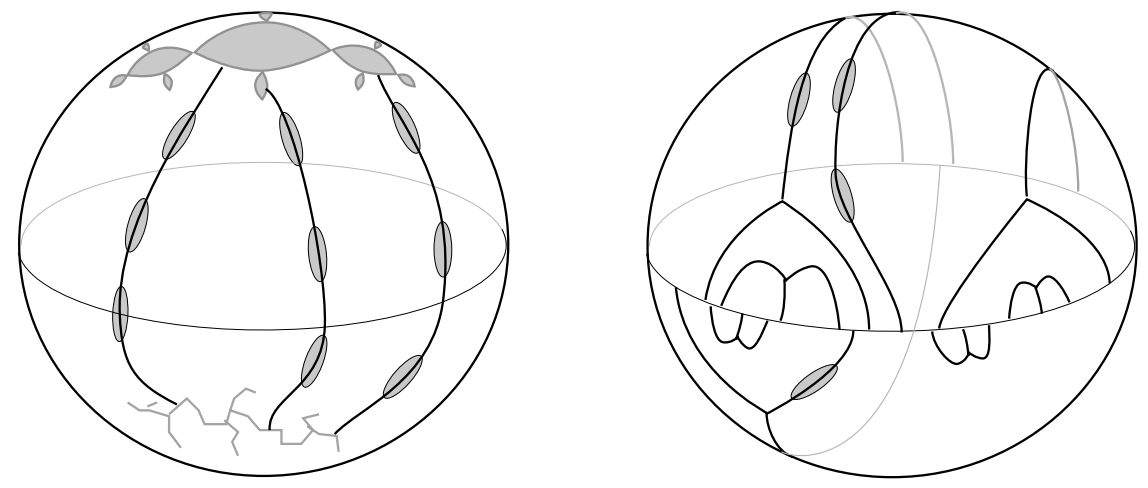

Figure 1. - On the left, we see a sphere $\widetilde{\mathbb{C}}_{1} \cup \widetilde{\mathbb{C}}_{2}$, with sketches of Julia sets and external rays drawn in. On the right, we see a sphere $\overline{\mathbb{H}} \cup \overline{\mathbb{H}}^{*}$ with the horizontal trajectories of a $\Gamma$-invariant quadratic differential sketched in $\mathbb{H}$ and the vertical trajectories of $q^{*}$ sketched in $\mathbb{H}^{*}$. In both figures, we can consider a family of Beltrami forms $\mu_{K}$ with eccentricity $K$ and big axes of the infinitesimal ellipses aligned with the external rays on the left, and with the horizontal trajectories of $q$ and $-q^{*}$ on the right. The effect of conjugating by a solution of the Beltrami equation $\bar{\partial} f=\mu_{K} \partial f$ is to shrink these curves.

\subsection{The setting for hyperbolic manifolds}

We set $\mathbb{H}$ to be the upper half-plane, and $\mathbb{H}^{*}$ to be the lower half-plane.

Let $\Gamma$ be a torsion-free Fuchsian group, such that $X:=\mathbb{H} / \Gamma$ is a Riemann surface of finite type; then $\mathbb{H}^{*} / \Gamma$ is the conjugate surface $X^{*}$.

Choose $q \in Q(\mathbb{H})$ a $\Gamma$-invariant quadratic differential, coming from an integrable quadratic differential on $X$; then $q^{*}(z)=\overline{q(\bar{z})}$ defines a $\Gamma$-invariant quadratic differential on $\mathbb{H}^{*}$, the reflection of $q$ in the real axis.

In analogy with matings of polynomials, the sphere, with the measured foliation $F_{1}:=|\operatorname{Im} \sqrt{q}|$ on $\mathbb{H}$ and the measured foliation $F_{2}:=\left|\operatorname{Im} \sqrt{-q^{*}}\right|$ on $\mathbb{H}^{*}$, could be called the formal mating of $F_{1}$ and $F_{2}$ (as far as I know, this terminology is not used).

Now take $s, t \in[0,1)$ and consider the Beltrami form on $\overline{\mathbb{C}}$ given by

$$
\mu_{q, s, t}= \begin{cases}s \frac{\bar{q}}{|q|} & \text { in } \mathbb{H} ; \\ t \frac{-\bar{q}^{*}}{|q|} & \text { in } \mathbb{H}^{*}\end{cases}
$$

This Beltrami form corresponds to a field of ellipses on $\overline{\mathbb{C}}$ 
- of eccentricity $(1+s) /(1-s)$ in $\mathbb{H}$, with major axes aligned along the leaves of $F_{1}$, i.e., along the horizontal trajectories of $q$,

- of eccentricity $(1+t) /(1-t)$ on $\mathbb{H}^{*}$, with major axes aligned along the leaves of $F_{2}$, i.e., along the vertical trajectories of $q^{*}$.

The object of taking the horizontal and vertical trajectories of the same quadratic differential was to make the two foliations transverse on $X$; and conversely, if two measured foliations are transverse, they correspond to the horizontal and vertical trajectories of a quadratic differential.

Let $f_{s, t}: \overline{\mathbb{C}} \rightarrow \overline{\mathbb{C}}$ be a quasi-conformal map such that

$$
\bar{\partial} f_{s, t}=\mu_{q, s, t} \partial f_{s, t} .
$$

Roughly speaking, the limit of the $f_{s, t}$ as $s, t \rightarrow 1$ consists of collapsing the trajectories of $F_{1}$ in $\mathbb{H}$ and the trajectories of $F_{2}$ in $\mathbb{H}^{*}$ to points. In fact, one can define a "topological mating of measured foliations", analogous to the topological mating of manifolds. Consider the leaf spaces of $F_{1}$ and $F_{2}$; they are $R$-trees with an action of $\Gamma$, and there is a natural equivalence relation on their disjoint union; the quotient space might be called to topological mating of $F_{1}$ and $F_{2}$ (although again I don't believe that the terminology is used). Since these $\mathbb{R}$-trees are always locally connected (unlike the case of polynomials, where $K_{p}$ might not be), it is quite reasonable to view them as intermediate objects in the process of constructing double limits. Mahan [7] does this.

Here the question is whether, up to conjugacy, the group representations

$$
\rho_{s, t}: \Gamma \rightarrow \mathrm{PSL}_{2} \mathbb{C} \text { given by } \rho_{s, t}:(\gamma)=f_{s, t} \circ \gamma \circ f_{s, t}^{-1}
$$

have non-empty compact accumulation sets when $s, t$ both tend to 1 , and whether this set consists of a single point. If the accumulation set is compact and non-empty, we say that the measured foliations $F_{1}$ and $F_{2}$ are mateable.

If $\rho_{\infty}$ is an accumulation point of the representations $\rho_{s, t}$, there is a map $\gamma: \mathbb{R} \mathbb{P}^{1} \rightarrow \mathbb{C P}^{1}$ that maps the circle $\mathbb{R P}^{1}$ surjectively to the limit set of $\rho_{\infty}(\Gamma) \subset$ Aut $\mathbb{P}^{1}$, that semiconjugates the action of the Fuchsian group $\Gamma$ on $\mathbb{R P}^{1}$ to the action of the Kleinian group $\rho_{\infty}(\Gamma)$ on its limit set. Most often this limit set is the entire sphere; this will happen for instance when each of $F_{1}$ and $F_{2}$ has a dense leaf on $\mathbb{H} / \Gamma$. This is generic in the space of quadratic differentials on $\mathbb{H} / \Gamma$.

In this case, we see that again $\gamma$ is a Peano curve: again we can unwind the sphere like a ball of yarn and get the much simpler action of a Fuchsian group on $\mathbb{R} \mathbb{P}^{1}$. 


\section{Parallel answers to the questions}

For matings of polynomials, two of the most striking results are the following.

TheOREm 3.1. - (Rees-Tan Lei [14]) Two post-critically finite quadratic polynomials are mateable if and only if they do not belong to conjugate limbs of the Mandelbrot set.

Theorem 3.2. - (Yampolsky-Zakeri [16]) Let $\theta_{1}, \theta_{2} \in[0,1)$ be irrationals of bounded type. Then the polynomials $e^{2 \pi i \theta_{1}} z+z^{2}$ and $e^{2 \pi i \theta_{2}} z+z^{2}$ are mateable if and if $\theta_{1} \neq 1-\theta_{2}$.

It should be true that any pair of quadratic polynomials from nonconjugate limbs of the Mandelbrot set are mateable: computer pictures certainly suggest it. Theorems 3.2 and 3.1 would both be special cases. Very recent work of Dzmitry Dudko, following earlier work of Jiaqi Luo, gives hope that something along these lines can be proved; in particular, Dudko gives a definition of a mating when the Julia sets are not locally connected

The "parallel statement" for Kleinian groups is the double limit theorem:

THEOREM 3.3. - (Thurston) Two measured foliations $F_{1}, F_{2}$ are mateable if any third measured foliation can be made transverse to one of $F_{1}$ or $F_{2}$.

This is not quite the original statement of the double limit theorem [13] which involves sequences of pairs of points in Teichmüller space approaching the Thurston Boundary.

I do not know if in this generality the accumulation set of the $\rho_{s, t}$ above is always reduced to a single point; I believe this follows from the Ending Lamination Conjecture (ELC, now proved [1],[6], which asserts that a limit is determined by the topological type (in this case, that of a surface) and the end-data. The end-data is rather difficult to define in general, but when the foliations $F_{1}$ and $F_{2}$ have dense leaves, it is the measured laminations associated to $F_{1}$ and $F_{2}$, forgetting about the associated measures (which may not be unique). So the accumulation set should be a single point in this case. I don't yet understand the statement or the proof of the ending lamination conjecture, so I will say nothing more about it here. Neither will I speak of the work of Mahan Mj [7], which describes the geometry of limit sets of limits of Kleinian groups in a way very similar to the quotient $\bar{S}_{p_{1}, p_{2}}=S_{p_{1}, p_{2}} / \approx_{p_{1}, p_{2}}$ : I don't understand the work well enough.

But in some cases the existence and uniqueness of the limit is true (and I understand the proof), most crucially when $F_{1}$ and $F_{2}$ are the stable and 
unstable foliations of a pseudo-Anosov homeomorphism of a surface. The existence of the limit

$$
\lim _{s, t \rightarrow 1} \rho_{s, t}
$$

in this case is fairly easily shown to be equivalent to the existence of a hyperbolic structure on 3-manifolds that fiber over the circle with pseudoAnosov holonomy.

Neither do I know if the condition stated is necessary as well as sufficient. Note that it implies, by taking $F_{3}=F_{1}$ that $F_{1}$ and $F_{2}$ can be made transverse, and that is necessary, essentially for the same reason that the condition in theorem 3.1 is necessary: in both cases, there are essential closed curves on the formal mating that would collapse to points in the true mating (or on the topological mating).

\section{The proofs}

There are many similarities in the proofs in the two fields, particularly when the two sides of the dictionary are specialized to postcritically finite polynomials on the dynamical systems side, and 3-d manifolds that fiber over the circle on the Kleinian groups side.

In that case, on both sides either the rational function or Kleinian group exist, or there is a multicurve that is an obstruction.

Moreover, the existence in both cases comes down to finding a fixed point of a mapping in Teichmüller space (or a product of compactified Teichmüller spaces).

In the more general case, the only method that I know of to approach existence of matings is puzzle techniques (that is what Yampolsky and Zakeri use to prove Theorem 3.2; it is also what Dudko and earlier Luo used). These have no parallel in the Kleinian world. In the Kleinian world, the proof uses Beltrami forms aligned with the horizontal and vertical foliations that are allowed to degenerate, i.e., whose norms are allowed to tend to 1. This is very similar to slow mating; the beautiful movies of slow mating have not yet led to proofs, but may well do so in the future. For examples of slow matings, go to Arnaud Chéritat's web page

$$
\text { http://www.math.univ-toulouse.fr/ cheritat/Gall/galery.html, }
$$

and find the film "two mating polynomial Julia sets"; there are several others equally as wonderful. 
And on the other side of the dictionary, maybe puzzles do have analogs. For instance, Masaaki Wada has written the marvelous program Opti, which explores 2-generator Kleinian groups with parabolic commutator. The space of conjugacy classes of such discrete groups is a product of two copies of the Teichmüller space for the punctured torus. The program draws the parameter space of such groups; it isn't obvious how to do this. In the process it "paves" the space by pieces corresponding to the different geometries of fundamental domains: not so different from paving parameter space for polynomials according to the corresponding dynamical puzzle. Maybe such pieces could be used as some sort of parapuzzle pieces.

\section{Bibliography}

[1] Brock (J.), Canary (R.), Minsky (Y.). - The Classification of Kleinian Surface Groups, II: the Ending Lamination Conjecture, arXiv:math/0412006.

[2] Hubbard (J. H.). — Teichmüller theory, vol. I, Matrix Editions (2006).

[3] Hubbard (J. H.). - Teichmüller theory, vol. II, Matrix Editions, to appear.

[4] McMullen (C.). - Iteration on Teichmüller space, Invent.math. 99, p. 425-454 (1990).

[5] McMullen (C.). - Rational maps and Kleinian groups, In Proceedings of the International Congress of Mathematicians (Kyoto, 1990), p. 889-900. SpringerVerlag (1991).

[6] Minsky (Y.). - The Classification of Kleinian Surface Groups, I. Models and Bounds, Ann. Math., 171(1), p. 1-107 (2010).

[7] Mahan (Mj). - Cannon-Thurston Maps for Kleinian Groups, arXiv:math arxiv:1002.0996.

[8] Otal (J.-P.). - The hyperbolization theorem for fibered 3-manifolds, Translated from the 1996 French original by Leslie D. Kay. SMF/AMS Texts and Monographs, 7. American Mathematical Society, Providence, RI; Société Mathématique de France, Paris (2001).

[9] ReEs (M.). - A partial description of parameter space of rational maps of degree two: Part I, Acta Math. 168, p. 11-87 (1992).

[10] Sullivan (D.). - Quasiconformal Homeomorphisms and Dynamics, I. Solution of the Fatou-Julia problem on wandering domains, Annals of math 122, p. 401-418 (1985).

[11] Thurston (W.). - On the geometry and Dynamics of iterated Rational Maps, in Complex Dynamics: Families and Friends, Schleicher ed., AK Peters, p. 3-130 (2009).

[12] Thurston (W.). - Hyperbolic structures on 3-manifolds, I: Deformation of acylindrical manifolds, Annals of Math 124, p. 203-246 (1986).

[13] Thurston (W.). - Hyperbolic structures on 3-manifolds, II: Surface Groups and 3-Manifolds that fiber over the Circle, arXiv:math/9801045.

[14] LeI (T.). - Matings of quadratic polynomials, Ergod. th. and dyn. syst., vo. 12, p. 589-620 (1992).

[15] WAdA (M.). - Opti, http: \\delta.math.sci.osaka-u.ac.jp \OPTi

[16] Wada (M.). - Mating Siegel Quadratic Polynomials, JAMS 14, p. 25-78 (2000). 